\title{
93. Über die allgemeine Verbreitung und das massenhafte Vorkommen von Pinnularia Brauni var. amphicephala (A. Mayer) Hustedt in den mineralogen- azidotrophen Gewässern Japans.
}

\author{
Von Ken-itirô Negoro. \\ Botanisches Institut der Tokyo Universität \\ für Literatur und Wissenschaft. \\ (Comm. by K. FUjII, M.I.A., Nov. 12, 1941.)
}

Die Diatomeenflora der mineralogen-azidotrophen Gewässer Japans ist sehr charakteristisch. In diesen, durch einen hohen Gehalt an freien Mineralsäuren ausgezeichneten Gewässer, die als Seen, Teiche, Quellen, Bäche usw. in vulkanischen Gebieten unseres Landes hier und da liegen, werden nur einige Formen festgestellt: Pinnularia Brauni (Grun.) Cleve ${ }^{1)}$, Pinnularia Brauni var. amphicephala (A. Mayer) Hustedt, Pinnularia acoricola Hustedt, Eunotia septentrionalis Östrup, Eunotia laevistriata Hustedt etc. Von diesen Formen ist Pinnularia Brauni var. amphicephala. (A. Mayer) Hustedt wegen ihrer weitesten Verbreitung und ihres massenhaften Vorkommens am auffallendsten.

Ich berichtete schon ihr Massenvorkommen in folgenden Orten: Kata-numa, ein Kraterssee bei Narugo, Miyagi Präfektur $(\mathrm{pH}$ des Wassers 1.4 $)^{2}$; Sikayu-gawa, ein Bach in den Sibukuro Thermalquellen, Akita Präfektur ( $\mathrm{pH} 1.7$, Temperatur des Wassers $\left.40.0^{\circ} \mathrm{C}\right)^{3}$; Okama,

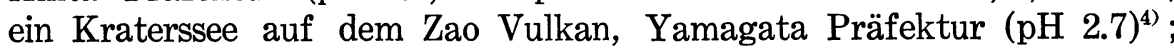
Itibisinai-ko, einer der Kratersseen in der Kunasiri Insel der Kurilen $(\mathrm{pH} 2.8)^{5}$.

In den letzten zwei Jahren machte ich unter finanzieller Unterstützung der kaiserlichen Akademie eine Reihe von Forschungsreisen nach den mineralogen-azidotrophen Gewässern vieler Gegenden unseres Vaterlandes. Meine Reisen erstreckten von Hokkaidô bis zu Kyûsyû und als Folge wurden wertvolle Ergebnisse gewonnen. Dafür möchte ich an dieser Stelle der Akademie meinen verbindlichsten Dank abstatten.

Durch diese Reisen erkannte ich, dass Pinnularia Brauni var. amphicephala als Massenform noch mehr in folgenden Gebieten auftritt. Die Temperatur und das $\mathrm{pH}$ des Wassers als Lebensraum der Diatomee, die bei den Untersuchungen an Ort und Stelle gemessen werden, sollen mit jedem Fundort unten gegeben werden :

1) Pinnularia Brauni (Grun.) Cleve tritt nur lokal und vereinzelt auf.

2) Bot. \& Zool., 6, (1938).

3) Bot. \& Zool., 8, (1940).

4) Jap. Journ. Limnol., 9, (1940).

5) Bot. Mag., 54, (1940). 
Fundort

I) Ôwaki-dani Solfatara im Temperatur des
Wassers $\left({ }^{\circ} \mathrm{C}\right)$ Hakone Gebirge, Kanagawa Präfektur.

1) Bächlein

2) Thermalquelle

23.8

34.2

2.8

2.1

II) Zigoku-numa, ein kleiner See bei den Sukayu Thermalquellen auf dem Hakkôda Berg, Aomori Präfektur.

3) See

4) Abflusschen des Sees...

III) Gosiki-numa Seengebiet am Fusse des Bandai Vulkans, Hukusima Präfektur.

5) Bisyamon-numa (See)

6) Aka-numa (See)

IV) Bach im Grundstücke eines Schwefelwerks bei Numajiri, Hukusima Präfektur.

7) Bach

V) Kata-numa, ein Kraterssee bei Narugo, Miyagi Präfektur.

8) See

9) Rinnsal am Ufer $(\alpha)$...

10) Rinnsal am Ufer $(\beta)$...

VI) Solfataren und Thermalquellen auf dem Osoresan Berg, Aomori Präfektur.

11) Sin-taki-no-yu (Thermalquelle)

12) Tümpel bei der obengenannten Thermalquelle

18.1

5.0

19.9

3.5

2.8

20.8

2.9

26. VII. 1940

18.1

29. VII. 1940

22.5

22.0

23.0

1.7

2.9

1.7

32.0

2.9

18. VII. 1941

26.0

2.2

35.0

2.4

32.5

1,7

31.0

2.6

24.0

2.6

28.0

32.2

Tag der

Untersuchung

30. V. 1940

28. VII. 1940

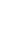


No. 9.] Über die allgemeine Verbreitung und das massenhafte Vorkommen.

\section{Fundort}

VIII) Zigoku-dani Solfatara bei den Noboribetu Thermalquellen, Hokkaidô.

20) Bach

21) Thermalquelle

IX) Sai-no-kawara Solfatara im Nasu Gebirge, Totigi Präfektur.

22) Rinnsal (a) ...............

23) Rinnsal ( $\beta$ )

24) Rinnsal ( $\gamma$ ) in unmittelbarer Nähe des berühmten Sessyô-Steins

X) Hatiman Solfatara und deren Umgebung auf dem Unzen Berg, Nagasaki Präfektur.

25) Bächlein ..................

26) Bach .......

27) Tümpel

28) Rinnsal

XI) Daikyôkan Solfatara und deren Umgebung auf dem Unzen Berg, Nagasaki Präfektur.

29) Bächlein

30) Nasser Boden der Schlammvulkane ......

XII) Kozigoku Solfatara und deren Umgebung auf dem Unzen Berg, Nagasaki Präfektur.

31) Nasser Boden

XIII) Zigoku Thermalquellen auf dem Aso Vulkan, Kumamoto Präfektur.

32) Moto-yu (Thermalquelle)

33) Suzume-no-zigoku (Thermalquelle)

XIV) Beppu Thermalquellen, Ôita Präfektur.

34) Rinnsal in einer Solfatara bei den Myôban Thermalquellen

35) Umi-zigoku (Thermalquelle) $\begin{array}{cc}\text { Temperatur des } & \mathrm{pH} \text { des } \\ \text { Wassers }\left({ }^{\circ} \mathrm{C}\right) & \text { Wassers }\end{array}$

17.0

32.0

2.8

21.7

24.3

19.0

46.2

32.4

33.5

34.0

45.7

22.0

31.9

26.7

26.0

31.0

48.0
2.8

1.7

2.6

3.2

19. VIII. 1941

2.0

2.9

2.8

4.0

Tag der

Untersuchung

23. VII. 1941

14. VIII. 1941

2.9

19. VIII. 1941

"

"

$"$

19. VIII. 1941

”

20. VIII. 1941

3.0

22. VIII. 1941

2.8

Die besonders prachtvolle Massenentwicklung wurde in den Orten Nr. 1, 2, 3, 8, 9, 10, 15, 16, 17, 18, 22, 23, 24, 26, 30, 32 und 33 beobachtet. Die Diatomee tritt in sehr grosser Individuenzahl und zwar als dunkel- oder hellbraune Überzüge an der Oberfläche der Steine oder des Schlamms fast rein auf. 
Wie oben ersichtlich ist, liegt die obere Temperaturgrenze der betreffenden Pflanze bei $48.0^{\circ} \mathrm{C}$. Aber in einer solchen hohen Temperatur (Nr. 22, 26 und 35) wächst sie - vergesellschaftet mit einer Cyanophycee, nämlich Cyanidium caldarium (Tilden) Geitler - nur vereinzelt. Der Temperaturbereich ihres günstigen Lebens scheint unterhalb $35^{\circ} \mathrm{C}$ $\mathrm{zu}$ liegen. Über $35^{\circ} \mathrm{C}$ hinaus überliess sie im allgemeinen dem Cyanidium caldarium ihren Platz.

Ihre Lebensbezirk im pH-Bereich liegt in dem untersuchten Material von 5.0 bis zu 1.7 (1.4). Dass sogar so hohe H-Konzentration wie $\mathrm{pH}$ 1.7 noch keinen nennenswerten, hemmenden Einfluss auf der Entwicklung dieser Diatomee ausübt, ist eine überraschende Tatsache.

Pinnularia Brauni var. amphicephala ist also eine Leitform in den mineralogen-azidotrophen Gewässern Japans. Sie ist jedoch in ihrem Vorkommen nicht auf solchen Gewässern beschränkt, sondern lebt stellenweise auch in neutralen oder selbst in alkalischen Gewässern. $\mathrm{Ob}$ sie auch in schwach gepufferten humussauren, sog. organogen-azidotrophen Gewässern wächst, ist mir fraglich, da ich sie bis heute aus den Hochmooren Japans noch nicht finden kann.

Es ist besonders lehrreich, dass ich sie neuerdings in einigen Salzgewässern unseres Binnenlandes finden konnte, dort sie beim $\mathrm{pH}$ 6.77.3 in Gesellschaft mit anderen, vielen mesohaloben und halophilen Diatomeen vereinzelt oder häufig auftritt. Ziemlich viele Diatomeenarten sind gegen hoch salziges Wasser widerstandfähig, aber die meisten von diesen Arten können die hohe H-Konzentration des Milieus gar nicht aushalten. Pinnularia Brauni var. amphicephala ist wahrlich eine der wenigen Diatomeenarten, die diese zwei abnormen Standortfaktoren gut ertragen können. Diese Eigenschaft erlaubt ihr, so denke ich, das Leben in den mineralogen-azidotrophen Gewässern.

Dürfen wir aus dem regelmässigen Auftreten und der massenhaften Entwicklung von 'Pinnularia Brauni var. amphicephala in den mineralogen-azidotrophen Gewässern trotz anderweitiger Verbreitung auf optimale Lebensbedingungen dieses Lebensraums schliessen? Kulturversuche können diese Frage lösen, doch vermute ich ihr Massenentwicklung in den mineralogen-azidotrophen Gewässern lediglich eine Folge der fehlenden Konkurrenz zu sein. Gleiche Ansicht vertrat Fr. Hustedt über die Ökologịe einiger Solfataren-Diatomeen ${ }^{1}$.

Fr. Hustedt untersuchte die Diatomeen aus den Solfataren von Mitteljava and Nordsmatra und feststellte 3 Arten, nämlich Eunotia fastigiata, Eunotia laevistriata und Pinnularia acoricola mit ihrer var. lanceolata als Leitformen dieses Biotops ${ }^{2}$. Pinnularia Brauni var. amphicephala tritt gar nicht in diesen mineralogen-azidotrophen Gewässern der Sunda-Inseln auf. Das allgemeine und massenhafte Vorkommen von Pinnularia Brauni var. amphicephala ist daher eine der bemerkenswerten Eigentümlichkeiten der Vegetation der mineralogenazidotrophen Gewässer Japans.

1) Arch. f. Hydrobiol., Suppl.-Bd. 15 (1938) u. 16 (1938).

2) Ebenda. 